\title{
Pervasive Agricultural Environment Monitoring System Based on Embedded Database
}

\author{
Hu Zhao, Sangen Wang ${ }^{* *}$, and Dake Wu \\ Southwest University, tiansheng road 216, chongqing, China \\ tiger100118@163.com, \{wangsg,keda5\} @swu.edu.cn
}

\begin{abstract}
According to space constraints of the traditional agricultural environment monitoring system, a small database called SQLite was transplanted into the ARM and Linux operation which could store and manage the field information. Users can get information anytime and anywhere through the GSM (Global System for Mobile Communications) network and the WSN(Wireless Sensor Network) by this design, instead of inquiring data from the PC(Personal Computer) which was widely used now. Sensors embedded in the environment, information spread in the air and captured by the handheld instruments, which is a typical application of pervasive computing.
\end{abstract}

Keywords: The pervasive computing, SQLite, WSN, ARM, GSM.

\section{Introduction}

Wireless sensor network of the field monitoring was constructed by Han Huafeng(2009) to transmitting the field acquired data to the designated database server on PC based on the ZigBee protocol, and data warehousing and decision support was used at the national agriculture statistics service by Yost M(2000). The most recently study was the WSN(Wireless Sensor Network) system based on the in-field soil water content monitoring designed by Jackson T, Mansfield K, Saafi M and so on(2007). Data of field were transmitted to the monitoring center through the GPRS network or Internet, and processed by the database system on PC in these systems. The field or greenhouse could be monitored remotely by the operator only in the monitoring center. These systems were expensive and complicate. So they were used only by universities and research institutes, but not in the vast rural areas.

The era of pervasive computing is coming with the development of technology. People can get information anytime and anywhere by using a handheld device. It has already been used widely in the Mobile TV, 3G phones and so on. In this article, a pervasive agricultural environment monitoring system based on embedded database was proposed to achieve the following objectives:

\footnotetext{
* Project supported by "the Key Program of Chongqing Natural Science" (CSTC2009BA1006) and "the Fundamental Research Funds for the Central Universities" (Grant No. XDJK2009C141).

** Corresponding author.
} 
(1) The greenhouse environmental data are transmitted to control module through WSN;

(2) The embedded system can collect, store and process the data independently;

(3) The system can send SMS to remind the users automatically at appointed time or the moment of abnormal;

(4) Users can query real-time and history data anytime and anywhere through mobile phones.

\section{Hardware Design}

The system is composed of control module and monitor units. The control module include MCU (Microprogrammed Control Unit), store module, GSM module and RF module; The real-time environmental information is detected and transmitted to the control module by monitor units scattered around the greenhouse. Structural diagram of the system is shown in Figure 1.

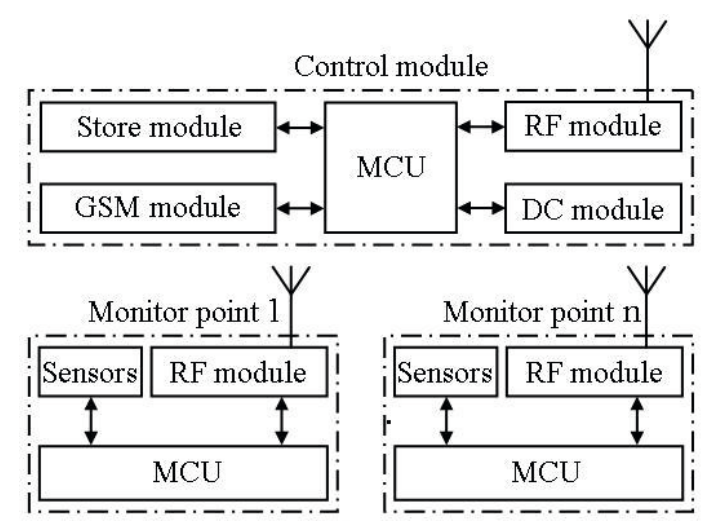

Fig. 1. Structural diagram of the system

The S3C2410 is used to manage data and control other modules in the control module. The NOR FLASH of $16 \mathrm{MB}$ is the program memory of the system with the read-write file system. Environmental data will be protected in the FLASH when the power is off suddenly. There are 6 Banks in the S3C2410, and 2 of them were assigned to the ROM, SRAM and SDRAM respectively. The SDRAM of the system is 2 HY57V561620CT of 32M bytes. The TC35 of Siemens works as SMS Module, and the RFM12 transmits and receives data as the wireless modules.

The monitors units include the MCU, sensors and the RF module. They spread around the greenhouse detecting the environmental information. The core of monitor units is a PIC16F877A with the strong anti-interference ability and rich resources. 


\subsection{Control Module}

The ARM processor has becoming the preferred choice for the embedded system design now. The S3C2410 is selected as the MUC considering the factors of the processing speed, storage space, interface and the power consumption. The core of the S3C2410 is an ARM920T and it can save cost and improve the performance with advantages of low prices, high performances and high level of integration. The DS1302 provide accurate date and time.

\subsection{WSN Module}

The star-type network topology was adopted for the WSN module. The Unique ID was corresponded to the different monitoring units. A number of monitoring units spread around the greenhouse which sent one packet per second. The control module received data by identifying the ID code circularly. For example, the control module received and processed the data of the second units only after receiving the first by identifying the ID code, and so on. The architecture of the WSN system is shown in Figure 2.

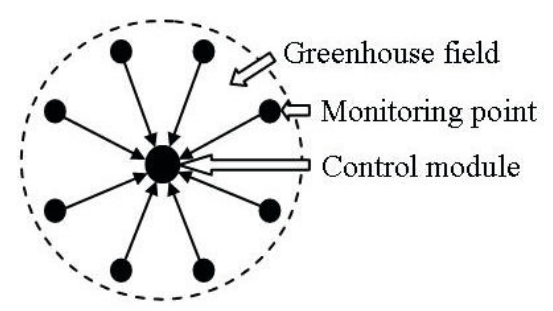

Fig. 2. Architecture of WSN system board

The RFM12 is a low costing ISM band transceiver RF module implemented with unique PLL. Its work signal bands ranges from 315/433/868/915MHZ, complying with FCC, ETSI regulations. The SPI interface is used to communicate with the MCU for the parameters setting. The data are sent into internal registers of RFM12 at the rising edge of the clock when the chip selecting signal nSEL is low. The synchronization header is sent at first, and then the data needed are sent. The next data can be sent when the SDO pin is high. The nIRQ pin is pulled low to notice the MCU when the RFM12 receives data. The MCU then reads the data stored in the buffer temporarily. The receiving register is closed when the receiving procedure finish.

The greenhouse's information includes the air temperature and humidity, soil temperature and humidity, light intensity and $\mathrm{CO}_{2}$ concentration. Digital sensors have the priority because of the calibration-free and high precision. It also makes it easy for multisensor fusion. The main performances of sensors are described as following: 
(1) Air temperature and humidity sensors-Used to detect the air temperature and humidity, and the model is the SHT10(Basic type), the main performances: the humidity measuring range $0 \sim 100 \% \mathrm{rh}$, temperature measuring range $-40 \sim$ $+123.8^{\circ} \mathrm{C}$, Temperature accuracy $\pm 0.3^{\circ} \mathrm{C}\left(25^{\circ} \mathrm{C}\right)$, humidity accuracy $\pm 1.8 \% \mathrm{RH}$, Response time $8 \mathrm{~s}$, power supply $2.4 \sim 5.5 \mathrm{~V}$, and the Digital output(I2C bus).

(2) Soil temperature and humidity sensors-Used to detect the soil temperature and humidity, and the model is the SHT10P(Protection type), the main performances: the humidity measuring range $0 \sim 100 \% \mathrm{rh}$; temperature measuring range $-40 \sim+123.8^{\circ} \mathrm{C}$; Temperature accuracy $\pm 0.3^{\circ} \mathrm{C}\left(25^{\circ} \mathrm{C}\right)$; humidity accuracy $\pm 1.8 \% \mathrm{RH}$; Response time $8 \mathrm{~s}$; power supply2.4 $\sim 5.5 \mathrm{~V}$; and the Digital output(I2C bus).

(3) Light intensity sensor-Used to detect the light intensity, and the model is the BH1710FVC. The main performances: the measuring range $0 \sim 65,535 \mathrm{Lx}$; Sensitivity $\pm 15 \%$; power supply $2.4 \sim 3.6 \mathrm{~V}$; and the Digital output (I2C bus).

(4) $\mathrm{CO}_{2}$ concentration sensor-Used to detect the $\mathrm{CO}_{2}$ concentration, and the model is $\mathrm{H}-550$. The main performance: the measuring range $0 \sim 5,000 \mathrm{ppm}$; sensitivity $\pm 30 \mathrm{ppm} \pm 5 \%\left(0 \sim 50^{\circ} \mathrm{C}\right)$; response time $\leq 30$ s; power supply DC $9 \mathrm{~V} \sim$ $18 \mathrm{~V}$; Current consumption $50 \mathrm{~mA}$ (mean value); and the Digital output(I2C bus).

\subsection{GSM Module}

TC35 is an industrial grade GSM module integrated RF modules and baseband processor.It Support data, voice, SMS and fax[8].The IGT pin maintain low(at least $100 \mathrm{~ms})$ at first when the TC35 start.Then it keep high to ensure TC35 work. Input/output interface of TC35 uses a serial asynchronous receiver, meet with ITUTRS232 interface standard. The data interface disposition is 8 bit data positions, one stop position, does not have the verification position. The hardware handshake signal use RTSO/CTSO.The software flow is controled by using Xon/Xoff. TC35 support AT command set.

\section{Software Design}

The software architecture of the data analysis unit is shown in Figure 3. The embedded operating system $\mu$ CLinux is located at the lowest layer. It manages the software and hardware resources of the system. The user programs are located at the most top layer. It receives and analyzes the environment parameters, and makes assess of the greenhouse' state. The database management system based on SQLite is located at core intermediate level. It is composed of the numerous program modules and the function is to organize, manage and deposit the shared data effectively.

\subsection{Design of Embedded Database System}

The $\mu$ CLinux is an open project funded and maintained by Lineo. It was made popular by its ability to run on processors without memory management hardware and with a typical kernel footprint of only 512 Kbytes or less. 


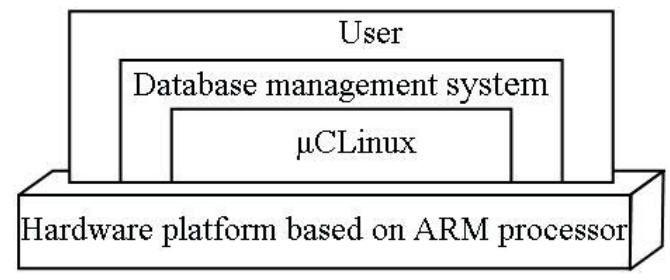

Fig. 3. Structural diagram of the data analysis unit

- It inherits stability and robustness from Linux;

- It supports a large number of devices. file systems, and networking protocols;

- No license fee leads to very low cost;

- It gives developers complete visibility of the source code based on GPL license;

- Kernel can be customized on demand.

The software platform was built on uClinux.

The system establishes cross compiling environment by using arm-elf-tools20030314.sh. This program is copy to the root directory first and run, then $\mu$ CLinux can be compiled and transplanted. A directory called "images" will be generated in the $\mu$ Clinux-dist/ directory after cross-compilation successfully. It includes 3 binary file: image.ram, image.rom and romfs.img.

The Bootloader is designed to make it sure that the execution jumps to the location of the kernel when resets. The MCU core must be initialized and extended in order to achieve the main functions. The whole procedure consists of the following steps:

(1) Establishing interrupt vector table;

(2) Initialization of various processor models;

(3) Introduction of special variables;

(4) Initialization of memory;

(5) Copy codes.

The right to use the CPU is returned to the operating system after hardware initialization process. Then the ultimate goal of the Bootloader is completed.

Structfile_operations structure is used to define the various operation set of equipment in the $\mu$ CLinux kernel. The main purpose of writing character device drivers is to achieve the various functions of structfile_operations structure. Structfile operations structure is defined in /include/linux/fs.h file. The drivers include the RF module, store module and GSM module. They were turned on and off by following functions:

(1) RF12_open()/RF12_close();

(2) FLASH_open()/FLASH_close();

(3) TC35_open()/TC35_close(). 


\subsection{Design of Embedded Database System}

Large amounts of environmental information are collected and processed in the system. The Database is needed to store and manage a large amount of data. Traditional enterprise databases such as Oracle, Sybase and so on have the difficult to work in the embedded system because of the limited resources. The SQLite is small, fast, and reliable, so it is a good choice for embedded systems. It has the following advantages compared with the traditional databases:

(1) It can run in the embedded devices whice have less memory and computing power;

(2) It can reset easily when the power or memory capacity runs out;

(3) The cost of establishment and maintenance is very low;

(4) It has strong portability to suit the complexity of embedded systems.

The SQLite is a universal embedded database. It must be transplanted and compiled before apply to the"S3C2410+uCLinux" environment. The main steps of transplanting are described below:

(1) Download sqlite-2.8.15.tar.gz;

(2) Unzip to directory uClinux-dist/user;

(3) User application settings.

The SQLite is added as a user application and converted into a shell. The following files needed to be compiled:

(1) uClinux-dist/user/Makefile

(2) uClinux-dist/config/Configure.help

(3) uClinux-dist/config/config.in

Run the program"make menuconfig" of uCLinux after above amendments. Selecte"CustomizeVendor/

User Settings" and "Miscellaneous Applications",

There will be a new"sqlite (NEW)" which added by us before. The SQLite will be compiled to parts of romfs at the stage of"make romfs" later.The following documents needed to be added and modified finally, and thus to complete the compilation of the SQlite under uCLinux.

- Modify sqlite/main.mk

- Add sqlite/Makefile

- Modify sqlite/src/os.c

- Modify sqlite/src/shell.c

The SQLite uses a standardized design structure based on relational database mode. It can be divided into 9 major auto units, as shown in Figure 4. These sub-functional units form the top-down tool chain: compile in the upper layer, query in the middle layer, storage data and operate system interface in the underlying.

The SQLite supports most of the standard SQL92 statement, especially to support the view, trigger and services. The API interface of the $\mathrm{C}$ language was provided by 
the SQLite insteading of the database engine. The access operations of data can be achieved by the way of calling the appropriate API functions directly. The Header file "sqlite3.h" must be referenced by the application program. There are 3 core API functions that should be emphasized:

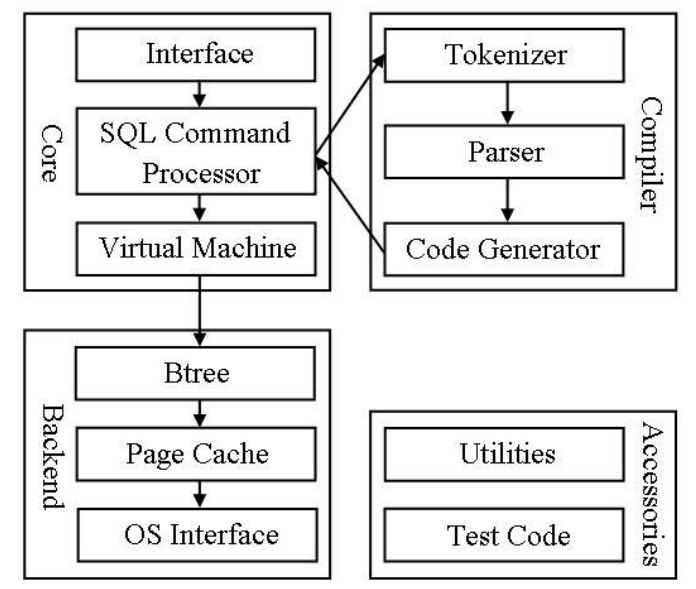

Fig. 4. Structural diagram of SQLite

(1) Open database

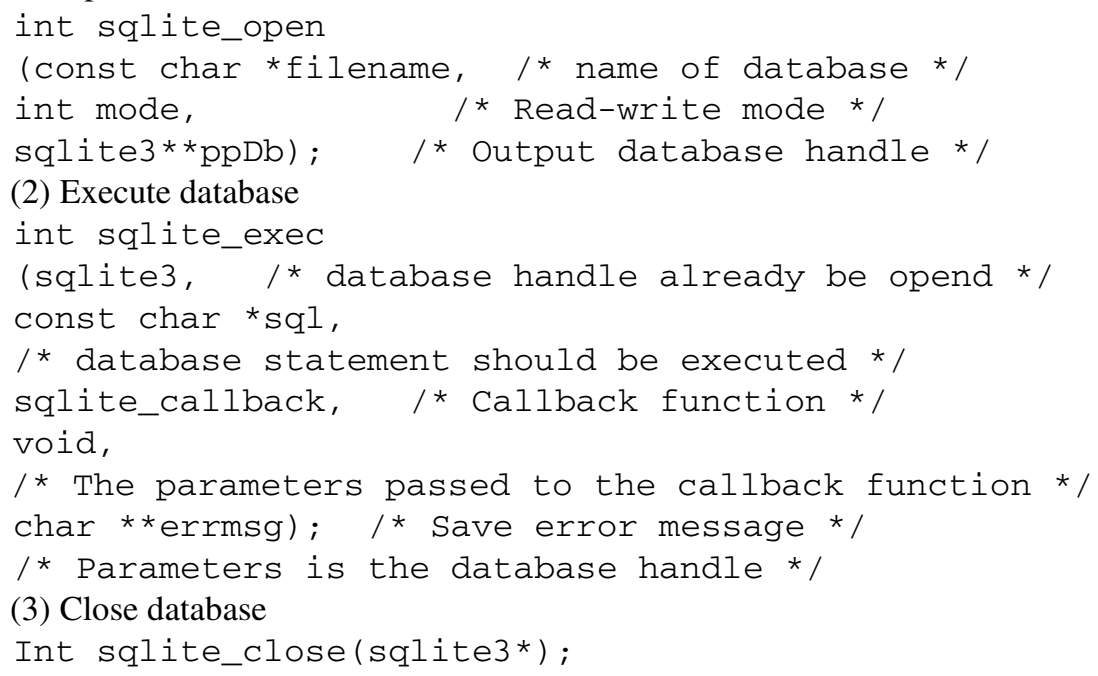

\subsection{Design of the Main Procedure}

The GSM module runes first when the main program begins. Receiver functions (read_sms()) are called to decoding the short message content when the SMS reaches (InterruptInt_Uart(void) interrupt 4 using 3()). The needed value is read from database 
and sent to the users(send_sms()). Then environmental information is received from the monitoring units(Receive_data()). The values are saved into database at the setting time. The alarm messages are sent to the users when the environmental values are exceeding the normal value. The flow chart of main procedure is shown in Figure 5.

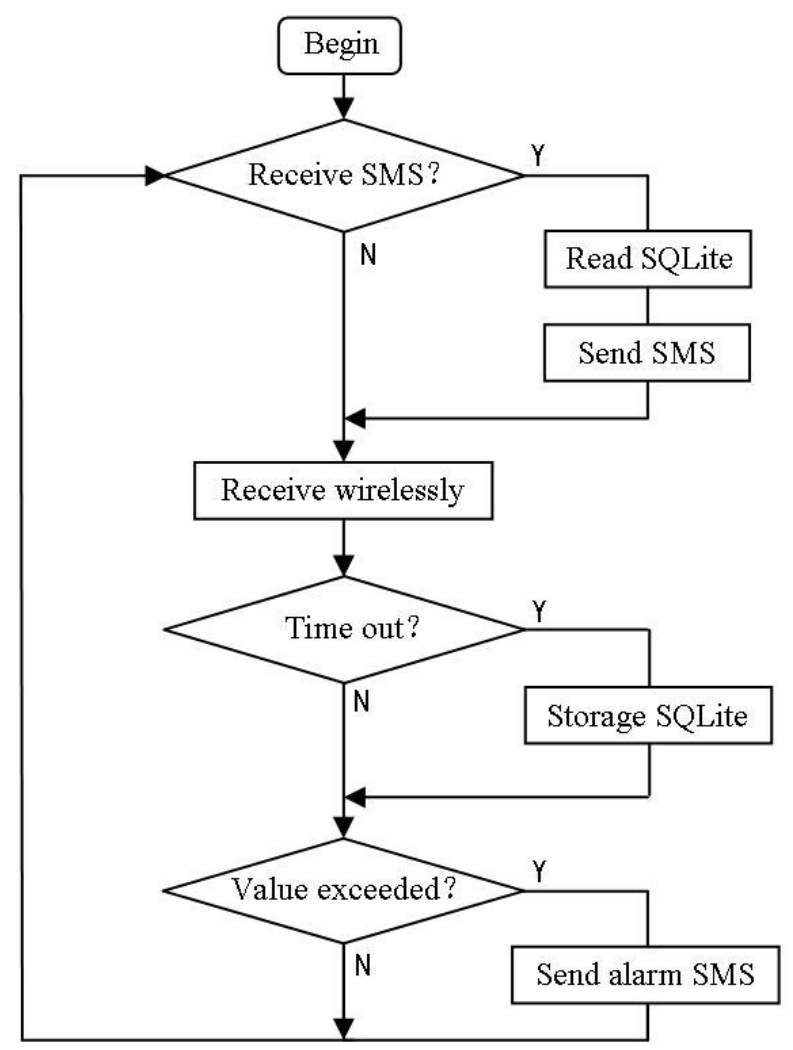

Fig. 5. Flow chart of main procedure

\subsection{Design of RF module}

The RFM12 is initialized (Open TX) at first in the sending mode, then calls subfunction "Send_data()" to send environmental parameters. The first two hexadecimal numbers of data are ID(Identify) codes which are unique for each unit. The next part of the data is the representatives of the air temperature and humidity, soil temperature and humidity, light intensity and $\mathrm{CO}_{2}$ concentration. The nIRQ pin is pull down at the end of sending to notice MCU sending the next data. The program diagram is shown in Figure 6(a). 
The RFM12 is initialized(Open RX) at first in the receiving mode, The nIRQ pin is pull down when the MCU reads the data. The FIFO must be reset after receiving the packet. The received data can be processed only through the validation procedures. The program diagram is shown in Figure 6(b).

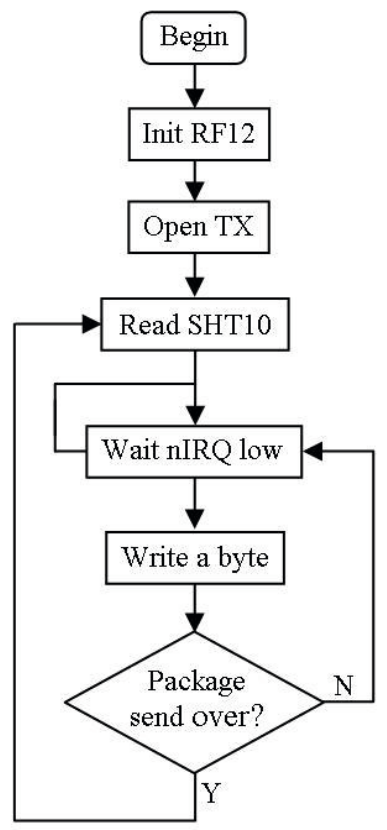

(a)

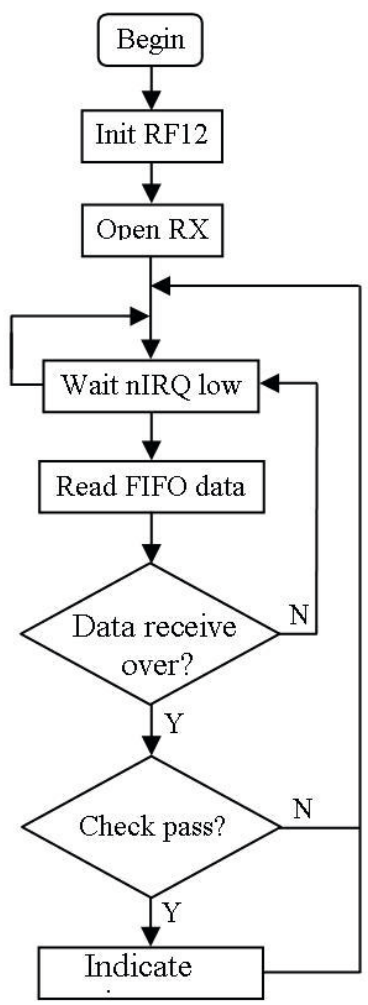

(b)

Fig. 6. Flow chart of RFM12

\section{Test Results}

The system was tested in the 3rd greenhouse of Southwest University agricultural demonstration center from October 2009 to May 2010. There are 16 monitoring units spreading evenly in the greenhouse. The monitored environmental parameters include the air temperature and humidity, soil temperature and humidity, light intensity and $\mathrm{CO}_{2}$ concentration. Data are automatically stored every 2 hours.

The SMS will be divided into two parts if the length is longer than 67 characters (including letters, numbers, or punctuation). The SMS query mode is designed as follows: 
(1) Send"201005040901",system will return" E24.53 ${ }^{\circ} \mathrm{C} 60.23 \% \mathrm{~S} 21.54{ }^{\circ} \mathrm{C} 72.36 \%$ Li31111lx C04237ppm" automatically, mean the parameters of 1st monitoring point are:air temperature $24.53^{\circ} \mathrm{C}$, air humidity $60.23 \%$,soil temperature 21.54 , soil humidity $72.36 \%$, light intensity $31111 \mathrm{~lx}, \mathrm{CO}_{2}$ concentration $4237 \mathrm{ppm}$;

(2) Send"date+te"(air temperature)," date+he"(air humidity)," date+ts"(soil temperature)," date+hs" (soil humidity)," date+li"(light intensity)," date+c02" $\left(\mathrm{CO}_{2}\right.$ concentration),system will return the data of the date.

Among the total of 521 text messages, there were 2 errors, 5 missing, and 514 corrects. The data could be store six months until the new covered them automatically. Experiments show that the greenhouse remote monitoring system based on"ARM + uCLinux + SQLite"performs reliably and efficiently.

\section{Conclusion}

The system that combined the pervasive computing with the agricultural information was suitable for the vast rural areas. The most significant features of the pervasive agricultural syetem are low cost, simple to use, and people-oriented design. It can be expected that control center room, wired communication network, and complex database system based on PC would disappear in the future. The farmers can monitor and control the environmental factors of the field or greenhouse anytime and anywhere by handheld devices.

\section{References}

1. Han, H., Du, K., Sun, Z., Zhao, W., Chen, R., Liang, J.: Design and application of ZigBee based telemonitoring system for greenhouse environment data acquisition. Transactions of the CASE, 158-163 (2009)

2. Yost, M.: Data warehousing and decision support at the national agriculture statistics service. Social Science Computer Renew, 434-441 (2000)

3. Gao, F., Yu, L., Zhang, W., Xu, Q., Yu, L.: Research and design of crop water status monitoring system based on wireless sensor networks. Transactions of the CASE, 107-112 (2009)

4. Li, Z., Wang, N., Hong, T., Wen, T., Liu, Z.: Design of wireless sensor network system based on in-field soil water content monitoring. Transactions of the CASE, 212-217 (2010)

5. Jackson, T., Mansfield, K., Saafi, M., et al.: Measuring soil temperature and moisture using wireless MEMS sensors. Journal of Measurement, 381-390 (2007)

6. Huisman, J.A., Sperl, C., Bouten, W., et al.: Soil water content measurements at different scales: accuracy of time domain reflectometory and ground-penetrating radar. Journal of Hydrology, 48-58 (2001)

7. Shen, L.: Pervasive Computing. Computer Engineering \& Science, 77-82 (2005)

8. Liao, D.: A Survey of the Pervasive Service. ZTE Communications, 32-35 (2008) 
9. Zeng, X., Liu, G., Zheng, D., Zhang, R.: Study development of a field information acquisition system based on wireless technique. Chinese Society of Agricultural Engineering Collected Papers, 151-154 (2005)

10. Li, X., Wang, W., Lei, T., Shen, Y.: Prospects of the application of multisensor information fusion techniques in agricultural engineering. Transactions of the CASE, 10-13 (2003)

11. Zhou, G., Zhou, J., Miao, Y., Liu, C.: Development and application on GSM-based monitoring system for digital agriculture. Transactions of the CASE, 158-163 (2009)

12. Hu, W.: Study on SQLite Implementation on the Embedded System. Computer \& Digital Engineering, 158-165 (2009) 